\title{
Green Communication for Mobile and Wireless Networks
}

\author{
Xiaohong Jiang, ${ }^{1}$ Han-Chieh Chao, ${ }^{2}$ Gabriel-Miro Muntean, ${ }^{3}$ \\ George Ghinea, ${ }^{4}$ and Changqiao $\mathrm{Xu}^{5}$
}

${ }^{1}$ Future University Hakodate, Hakodate, Japan
${ }^{2}$ National Ilan University, Yilan, Taiwan
${ }^{3}$ Dublin City University, Dublin, Ireland
${ }^{4}$ Brunel University London, London, UK
${ }^{5}$ Beijing University of Posts and Telecommunications, Beijing, China

Correspondence should be addressed to Xiaohong Jiang; jiang@fun.ac.jp

Received 27 July 2016; Accepted 28 July 2016

Copyright (C) 2016 Xiaohong Jiang et al. This is an open access article distributed under the Creative Commons Attribution License, which permits unrestricted use, distribution, and reproduction in any medium, provided the original work is properly cited.

Recent advances of various mobile communication devices have driven us into the era of smart society. Additionally, rapid development of wireless communication enables these devices to communicate with each other and exchange information to perform the optimal control or display necessary information ubiquitously. However, lacking of cooperation among mobile nodes results in not only a low communication quality but also imbalances of the resource utilization in mobile wireless networks and hence greatly increases the unnecessary energy consumption. Green communication such as information sharing, spectrum/energy awareness, routing adaptation, and data caching enables balancing the resource utilization and saving the energy of entire mobile and wireless networks. Therefore, green communication becomes the utmost important and promising research topic for future mobile and wireless networks.

This special issue intends to provide a collection of the latest researches in the field of green communication for mobile and wireless networks. We select nine papers after meticulous reviews and extensive discussions among Guest Editors and the Editor-in-Chief.

The paper titled "A Game-Theoretical Approach for Spectrum Efficiency Improvement in Cloud-RAN" proposed by $Z$. Zhou et al. considers the problem of remote radio heads (RRHs) mutual interference in cloud radio access networks (Cloud-RAN). The problem is formulated as a coalition formation game in partition form, which enables RRH to join and leave the coalition according to the individual utility as well as the coalition utility. Additionally, a hybrid multiple access mode based coalition is proposed to achieve the cooperative transmission among the RRHs. Based on these mechanisms, the intercell interference among the RRHs can be effectively mitigated and hence spectrum efficiency in terms of energy can be improved.

The paper titled "Social Optimization and Pricing Policy in Cognitive Radio Networks with an Energy Saving Strategy" by S. Jin et al. presents a novel energy saving strategy in cognitive radio networks (CRNs) and an appropriate pricing policy for secondary user (SU) packets. To evaluate the proposed methods theoretically, the system performance in terms of average latency of SU packets and energy saving ratio were analyzed by solving a quasi-birth-and-death process. Besides, the individually optimal behavior and the socially optimal behavior of SU packets are studied individually via rewardcost structure and compared. Based on the comparison, an appropriate admission fee is imposed on SU packets which aims to further improve the energy saving.

The paper titled "Interaction-Aware Video CommunityBased Content Delivery in Wireless Mobile Networks" by Y. Zhong et al. proposes a novel interaction-aware video community-based content delivery (IVCCD) method over mobile environment. A user interaction model is built to capture common characteristics in the behavior of request and delivery content. A partition-based community discovery scheme is presented by grouping the mobile users with common characteristics in order to provide high efficiency video 
sharing. Community member management and resource sharing scheme are also designed to optimize the community maintaining cost and accelerating resource lookup speed, which further support the green communications for mobile video streaming service. The paper titled "Modelling of P2P-Based Video Sharing Performance for Content-Oriented Community-Based VoD Systems in Wireless Mobile Networks" by S. Jia et al. constructs two models for the node state variation in terms of joining and quitting video system. According to the built models, the degrees of three factors which influence the video sharing performance, available upload bandwidths, resource distribution in overlay, and node mobility, are analyzed. Based on the analysis, the authors improve the communication between communities by the participation permission of available resource assignment. A service priority based incentive mechanism is proposed for optimal resource distribution. Combining with these two strategies, the control overhead and content delivery cost can be reduced and hence save the energy consumption of mobile video system.

The paper titled "Energy Efficient Interest Forwarding in NDN-Based Wireless Sensor Networks" by S. Gao et al. focuses on the energy efficient design issues over emerging network paradigm Named Data Network (NDN) and proposes a dual mode interest forwarding (DMIF) scheme. Series of mechanisms including flexible mode shift, flooding scope control, broadcast storm avoidance, packet suppression, and energy weight factors are designed in DMIF, which aims to save and balance the energy consumption in NDN-based WSNs.

In the paper titled "A Novel Energy Saving Resource Allocation Scheme in LTE-A Relay Networks" by Z.-Y. Chen, the authors discuss a resource allocation problem to minimize the total energy consumption of user equipment (UEs) and guarantee the constraints of QoS, bit-error-rate (BER), total system resource, and maximum transmit power. An efficient method to solve the problem is proposed and the time complexity of which has been proven to be acceptable. The article "Energy-Saving Management Mechanism Based on Hybrid Energy Supplies for LTE Heterogeneous Networks" by $\mathrm{P}$. Yu et al. proposes a novel energy saving (ES) management mechanism which considers hybrid energy supplies and selforganized network (SON). This mechanism divides the ES problem into four stages: traffic variation prediction, regional Base Station (BS) mode determination, BS-user association, and power supply. Four low-complexity algorithms for each stage are proposed, which aim to not only save the energy but also ensure the network performance.

In the paper titled "Reverse Auction Based Green Offloading Scheme for Small Cell Heterogeneous Networks" proposed by $\mathrm{X}$. Xu et al., the authors employ the reverse auction theory to handle the offloading decision process. Based on the reverse auction model, the energy efficiency optimization problem under the constraint of QoS, available bandwidth, and transmission power is modeled as dynamic programming and solved by Kuhn-Tucker conditions. The article titled "Technoeconomical Analysis of Macrocell and Femtocell Based HetNet under Different Deployment Constraints" by S. F. Yunas et al. compares the performances of two deployment strategies in terms of technoeconomic homogeneous macrocellular densification and a heterogeneous macro-femto deployment strategy. According to the comparison, the authors conclude that ultradense deployment of small cells together with larger bandwidth, mainly through carrier aggregation, will form an integral part of network evolution strategies, which achieves both cost efficiency and energy efficiency.

\section{Acknowledgments}

We would like to thank all authors who submitted their papers for this special issue and all reviewers for their feedback and comments on the articles. Their hard work and valuable contributions help us improve the quality of this special issue. Finally, we hope that these articles can provide a useful and informative reference to readers and be a stimulus for the further research on green communications over the mobile and wireless networks.

\author{
Xiaohong Jiang \\ Han-Chieh Chao \\ Gabriel-Miro Muntean \\ George Ghinea \\ Changqiao Xu
}



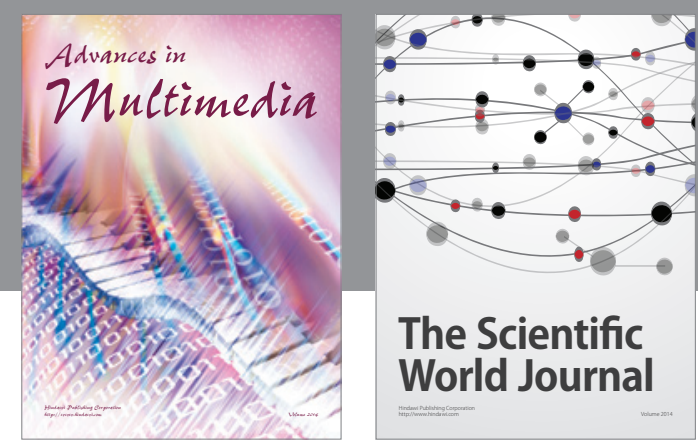

The Scientific World Journal
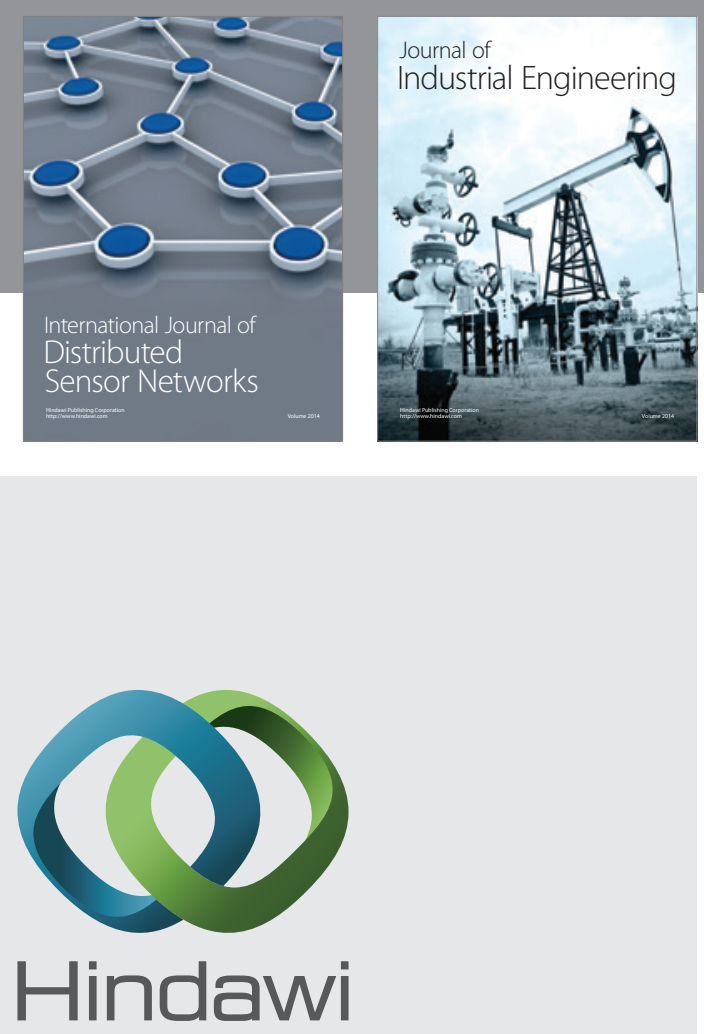

Submit your manuscripts at

http://www.hindawi.com

\section{Computer Networks} and Communications
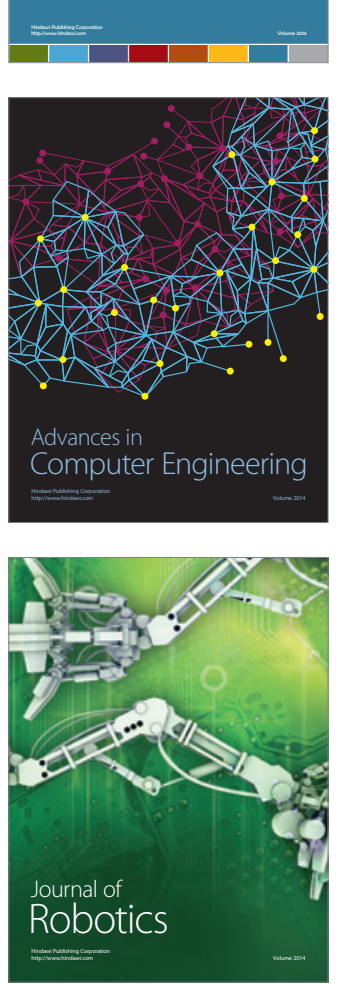
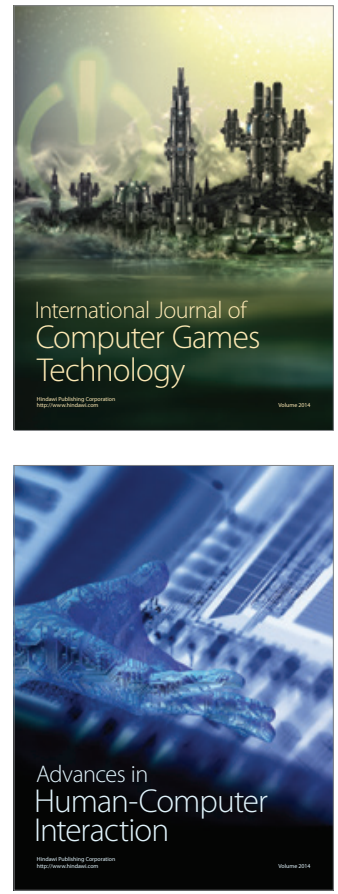
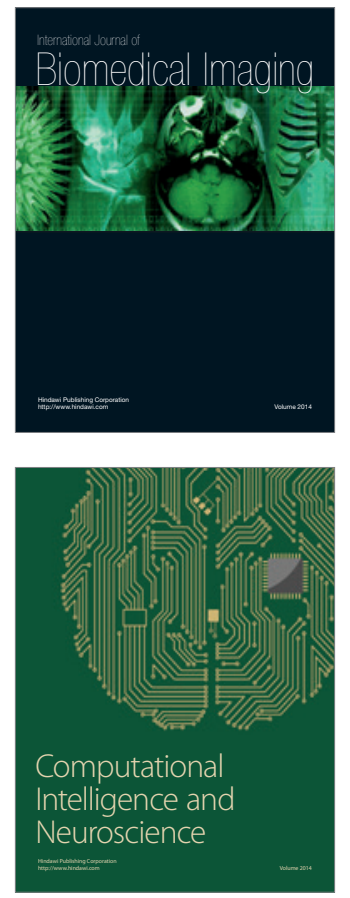
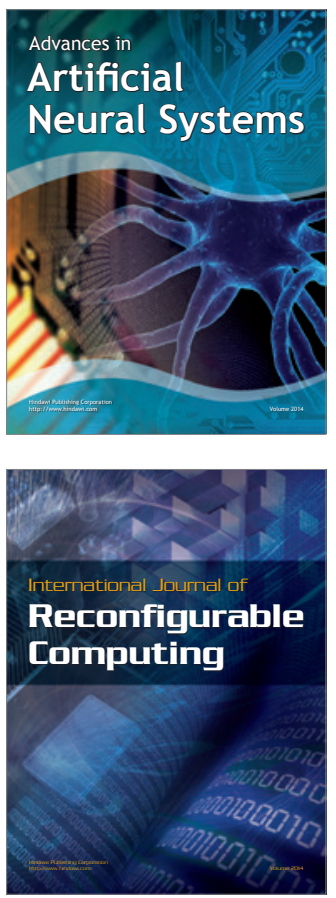
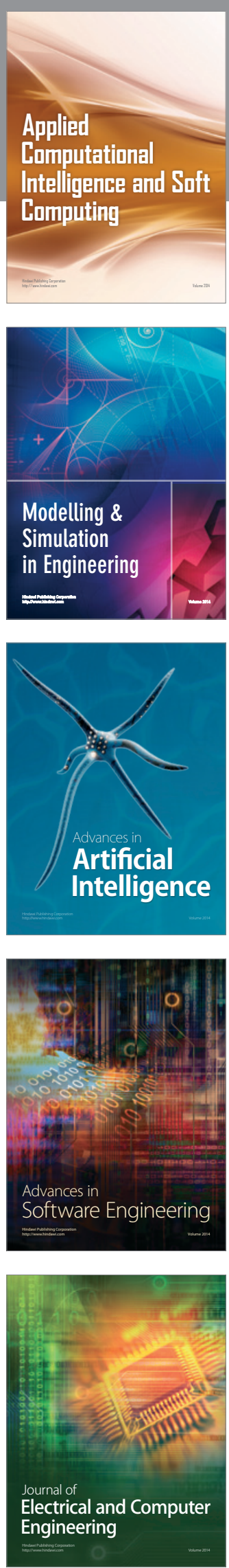